OPEN ACCESS

Edited by:

Jose Carlos Alves-Filho,

University of São Paulo, Brazil

Reviewed by:

Arpan Acharya,

University of Nebraska Medical Center,

United States

Kenichi Okuda,

University of North Carolina at Chapel

Hill, United States

*Correspondence:

Ricardo Sánchez-Rodríguez

ricardo.sanchezrodriguez@unipd.it

Barbara Molon

barbara.molon@unipd.it

${ }^{+}$These authors have contributed equally to this work

Specialty section: This article was submitted to Cytokines and Soluble Mediators in Immunity, a section of the journal

Frontiers in Immunology

Received: 22 September 2021 Accepted: 16 November 2021 Published: 07 December 2021

Citation:

Cioccarelli C, Sánchez-Rodríguez $R$, Angioni R, Venegas FC, Bertoldi N, Munari F, Cattelan A, Molon B and Viola A (2021) IL1 $\beta$ Promotes TMPRSS2 Expression and SARSCoV-2 Cell Entry Through the p38 MAPK-GATA2 Axis.

Front. Immunol. 12:781352. doi: 10.3389/fimmu.2021.781352

\section{IL1 $\beta$ Promotes TMPRSS2 Expression and SARS-CoV-2 Cell Entry Through the p38 MAPK-GATA2 Axis}

\author{
Chiara Cioccarelli ${ }^{1,2 t}$, Ricardo Sánchez-Rodríguez ${ }^{1,2^{*}+}$, Roberta Angioni ${ }^{1,2}$, \\ Francisca C. Venegas ${ }^{1,2}$, Nicole Bertoldi ${ }^{1,2}$, Fabio Munari ${ }^{1,2}$, Annamaria Cattelan ${ }^{3}$, \\ Barbara Molon ${ }^{1,2 *}$ and Antonella Viola ${ }^{1,2}$
}

${ }^{1}$ Department of Biomedical Sciences, University of Padova, Padova, Italy, ${ }^{2}$ Istituto di Ricerca Pediatrica (IRP), Fondazione
Città della Speranza, Padova, Italy, ${ }^{3}$ Infectious Disease Unit, Padova University Hospital, Padova, Italy

After the outburst of the SARS-CoV-2 pandemic, a worldwide research effort has led to the uncovering of many aspects of the COVID-19, among which we can count the outstanding role played by inflammatory cytokine milieu in the disease progression. Despite that, molecular mechanisms that regulate SARS-CoV-2 pathogenesis are still almost unidentified. In this study, we investigated whether the pro-inflammatory milieu of the host affects the susceptibility of SARS-CoV-2 infection by modulating ACE2 and TMPRSS2 expression. Our results indicated that the host inflammatory milieu favors SARS-CoV-2 infection by directly increasing TMPRSS2 expression. We unveiled the molecular mechanism that regulates this process and that can be therapeutically advantageously targeted.

Keywords: GATA2, SARS-CoV-2, IL1 $\beta$, p38, TMPRSS2

\section{INTRODUCTION}

At more than one year after the Coronavirus disease 19 (COVID-19) has been declared a global pandemic, the Severe Acute Respiratory Syndrome Coronavirus 2 (SARS-CoV-2) infection is still threatening the healthcare systems around the world. The critical and severe forms of COVID-19 are associated with pneumonia and Acute Respiratory Distress Syndrome (ARDS), conditions that are triggered by deregulated immunity and uncontrolled inflammation. In particular, the inflammatory cytokine milieu of the patient plays a crucial role in COVID-19 progression. Indeed, specific cytokines have been linked to worse severity, longer hospitalization time, or to patient's age (1). Accordingly, multiple diseases such as diabetes and obesity, as well as aging, that are characterized by chronic inflammation and elevated levels of pro-inflammatory cytokines, represent high risk factors for developing a severe illness after SARS-CoV-2 infection (2). Among the cytokines that have been associated to COVID-19 severity, several reports identified higher levels of IL1 $\beta$ in severe and critical patients compared with mild and moderate ones (3). Actually, SARS-CoV-2 infection is known to trigger the NLRP3 inflammasome activation in various cell types and the activation of this pathway, that leads to a strong increase in IL1 $\beta$ release, might exacerbate ARDS and systemic inflammation (4). 
SARS-CoV-2 entry into the target cells involves the binding of the viral spike protein (S) to the host Angiotensin I Converting Enzyme 2 (ACE2) receptor and its proteolytical activation by proteases. Following the binding of the S1 subunit to ACE2 through its receptor-binding domains (RBD), the host transmembrane protease/serine subfamily 2 (TMPRSS2) cleaves the $S$ protein to facilitate membranes fusion (5). The expression levels and the co-expression of ACE2 and TMPRSS2 seem to be key factors in determining the susceptibility of target organs to SARS-CoV-2 infection (6). Recently, it has been proved that ACE2 expression can be regulated in response to interferon alpha and gamma signaling (7), linking the immune response to the modulation of the cell receptors that mediate SARS-CoV-2 entry. TMPRSS2 is expressed in several tissues including lungs, gastrointestinal tract and kidney. Notably, the TMPRSS2 gene is upregulated in response to androgenic hormones (8), suggesting that the expression of this protease might be involved in the observed higher severity of COVID-19 in men compared to women.

\section{RESULTS AND DISCUSSION}

In this study, we investigated whether the pro-inflammatory milieu of the host affects TMPRSS2 and ACE2 expression in SARS-CoV-2 target cells. To this aim, the A549 human lung cell line was exposed to various concentrations of IL1 $\beta$, IL6, and TNF $\alpha$ inflammatory cytokines and the expression level of TMPRSS2 and ACE2 mRNA was evaluated. Interestingly, we found that IL $1 \beta$ and TNF $\alpha$ stimulation enhanced the transcription of TMPRSS2 (Figure 1A and Supplementary Figure 1A). On the other side, TNF $\alpha$ and IL1 $\beta$ treatment did not affect ACE2 expression that was rather increased by IL6 (Supplementary Figures 1B, C). The effect of IL1 $\beta$ over TMPRSS2 and ACE2 expression was also confirmed in primary immortalized human alveolar epithelial cells (TT1) (Figure 1B and Supplementary Figure 1D).

In addition to TMPRSS2, other proteases, such as ADAM17 and FURIN, have been reported to promote SARS-CoV-2 infection (9) but none of them appeared to be transcriptionally regulated by pro-inflammatory cytokine stimulation in the A549 cells (Supplementary Figure 2).

In line with other reports (4), our data confirmed that COVID19 patients showed an increased level of circulating IL1 $\beta$ (Figure 1C) compared to healthy subjects. Notably, IL1 $\beta$ concentration was significantly higher in severe and critical patients compared with mild and moderate ones (Figure 1D). Therefore, we focused our analysis on IL1 $\beta$-induced transcriptional regulation of TMPRSS2 expression. We first validated the induction of TMPRSS2 expression by IL1 $\beta$ stimulation at the protein level by performing immunofluorescence and western blot analyses on both A549 (Figures 1E-G and Supplementary Figure 3) and TT1 cells (Figures $\mathbf{1 H}-\mathbf{J}$ and Supplementary Figure 3). Furthermore, we evaluated whether IL1 $\beta$ stimulation can modulate SARS-CoV-2 endosome and replication relatedgenes such as IFITM2 (10) and RNF20 (11). No transcriptional changes were observed for these genes upon IL1 $\beta$ stimulation in lung cells (Supplementary Figure 4).

Previous studies showed that TMPRSS2 expression is controlled by the master regulator transcription factor GATA2 through its binding to the $-13 \mathrm{~kb}$ DNA enhancer region of the gene (-13 kb upstream the promotor) (8). Furthermore, GATA2 activation has been reported to be mediated by its phosphorylation via p38 MAPK pathway (12). GATA2 is also involved in the transcriptional regulation of several proinflammatory cytokines, including ILIB and CXCL2 (12). In addition, a positive feedback between IL1 $\beta$ signaling and GATA2 activation has been previously described in Acute Myeloid Leukemia (12). Thus, we speculated that IL1 $\beta$ may induce TMPRSS2 expression through the p38 MAPK-GATA2 axis. To validate our hypothesis, we first analyzed the effect of IL1 $\beta$ stimulation in A549 and TT1 cells in the presence or absence of specific inhibitors that selectively block p38 MAPK and GATA2 activity. Remarkably, we observed that TMPRSS2 transcription was significantly decreased by the treatment with p38 MAPK (Birb796) or GATA2 (K7174) inhibitors in IL1 $\beta$ stimulated A549 and TT1 cells (Figures 1K, L and Supplementary Figure 5A). ACE2 transcription was not affected by the inhibitors (Supplementary Figure 5B).

In order to confirm the role of GATA2 on the IL1 $\beta$-induced transcriptional regulation of TMPRSS2, GATA2 chromatin immunoprecipitation was performed. Our results showed that the chromatin enrichment in the TMPRSS2 -13 kb enhancer region was increased upon IL1 $\beta$ stimulation (Figure $\mathbf{1 M}$ ). Importantly, the p38 MAPK and GATA2 inhibitors, that blocked TMPRSS2 overexpression induced by IL1 $\beta$, were also able to inhibit the GATA2 binding to the enhancer region (Figure 1M). Moreover, GATA2 transcription was not affected by pro-inflammatory cytokine exposure (Supplementary Figure 6), suggesting that the outlined effect relies on GATA2 activity rather than on a modulation of its expression level. To further corroborate the role of GATA2 on TMPRSS2 transcription upon IL1 $\beta$ stimulation, we performed GATA2 silencing in A549 cells. Our results confirmed that the downregulation of GATA2 expression blocked the enhancement of TMPRSS2 transcription in response to IL1 $\beta$ exposure. (Figure 1N and Supplementary Figures 7A-C).

Finally, we evaluated whether the IL1 $\beta$-induced TMPRSS2 overexpression could increase cell susceptibility to SARS-CoV-2 infection. A549 and TT1 cells were exposed to the nonreplicative heat-inactivated SARS-CoV-2 virus, in presence or absence of IL1 $\beta$ and of the selected inhibitors. We observed that IL1 $\beta$ exposure increased the virus entry (Figures 1O-R and Supplementary Figures 7D, E), which was significantly inhibited by the pharmacological block of the p38 MAPKGATA2 axis. IL $1 \beta$ has been reported to promote the expression of interferon response genes in epithelial and myeloid cells (13). We found that IL1 $\beta$ stimulation promoted the overexpression of IRF1 (Supplementary Figures 8A, B) and its target (14) ISG15 (Supplementary Figures 8C, D) but not of IRF9 (Supplementary Figures 8E, F) in lung cells. Of note, the expression of these targets has been associated with cytokine 

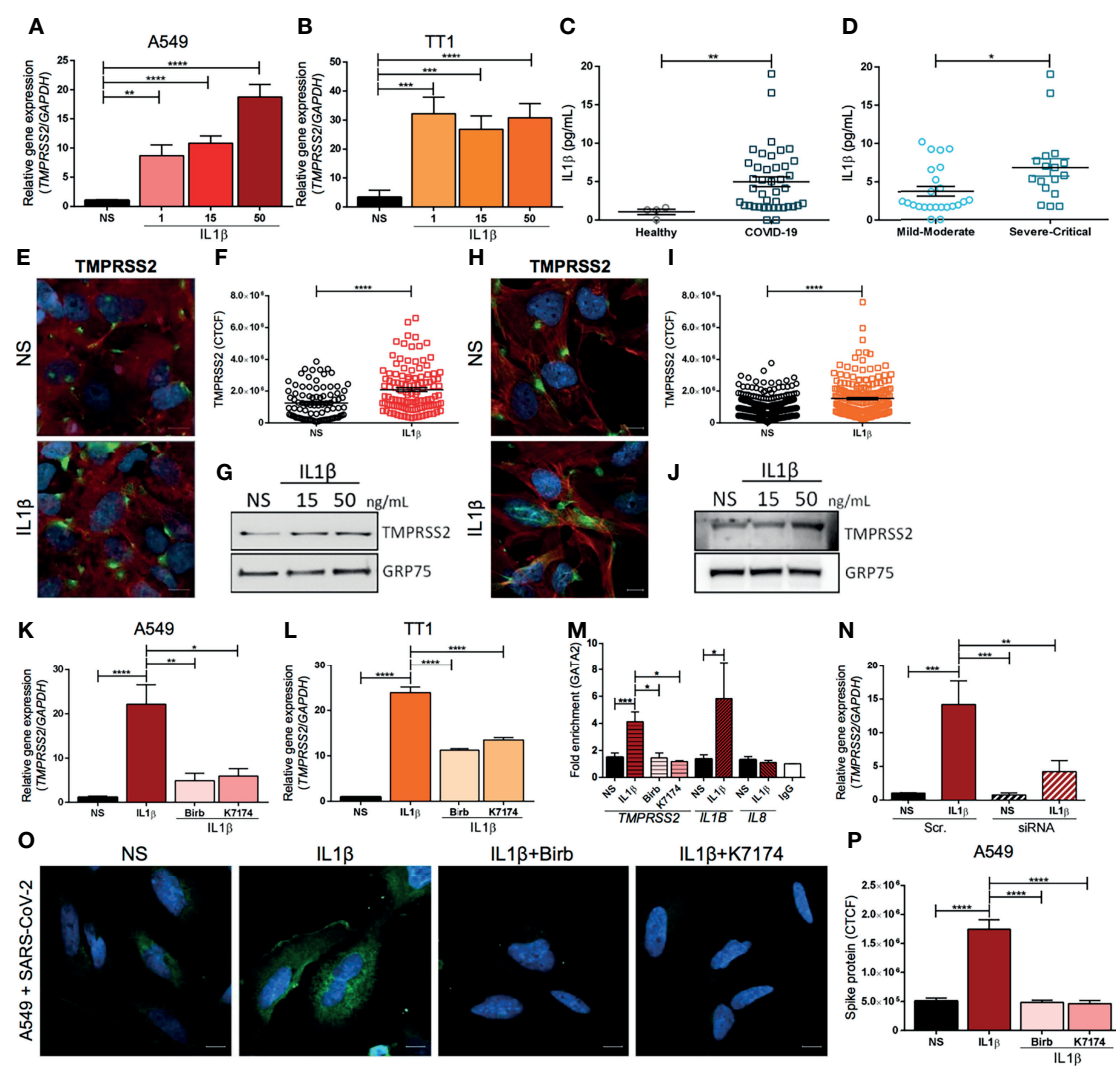

IL $1 \beta+K 7174$
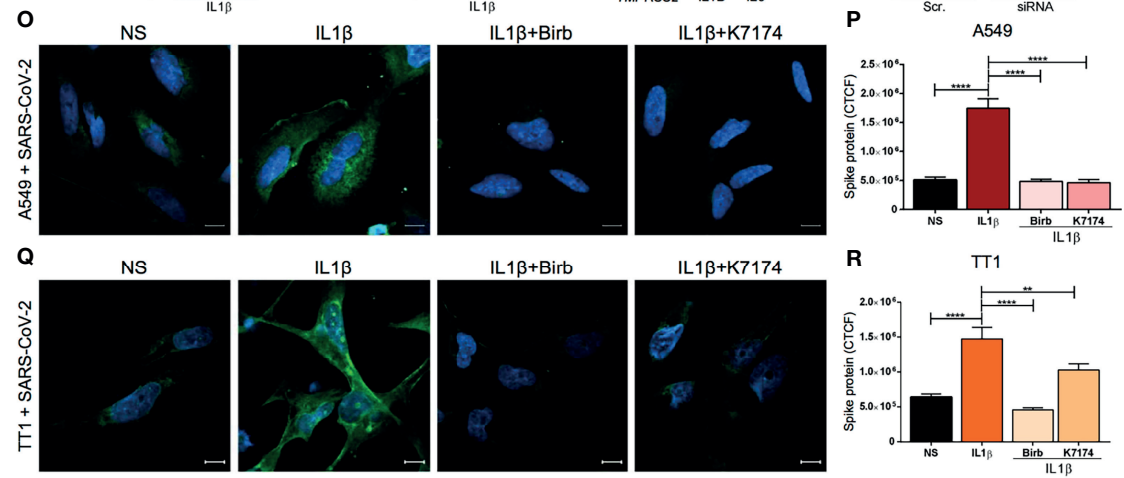

IL1 $\beta+K 7174$

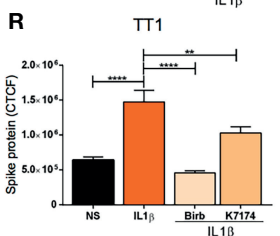

FIGURE 1 | TMPRSS2 expression is up-regulated by inflammatory cytokines enhancing SARS-CoV-2 entry in human lung cells. RT-qPCR analysis of the relative gene expression of TMPRSS2 in (A) A549 and (B) TT1 cells either stimulated or not (NS) for 4 hours with IL $1 \beta$ (1, 15 or $50 \mathrm{ng} / \mathrm{mL}$ ); $\mathrm{n}=5 \mathrm{independent} \mathrm{experiments.}$ IL1 $\beta$ plasma concentration (pg/mL) (C) in healthy, age-matched controls and COVID-19 patients, and (D) in COVID-19 patients grouped by disease severity (mildmoderate versus severe-critical); $n=44$ patients. (E) Representative immunofluorescence for TMPRSS2 and (F) quantification of fluorescence intensity of TMPRSS2 (green) in A549 cell line either stimulated or not (NS) for 24 hrs with IL $1 \beta$ (50 ng/mL). Nuclei were counterstained with Hoechst 33342 (blue) and actin was stained with Phalloidin TexasRed (Red). CTCF: corrected total cell fluorescence. Scale bar: $10 \mu \mathrm{m} ; \mathrm{n}=3$ independent experiments. (G) Western blot analysis of TMPRSS2 in total protein lysates from A549 cells stimulated or not (NS) for 24 hours with IL1 $\beta$ (15 or $50 \mathrm{ng} / \mathrm{mL}$ ); GRP75 was used as a loading control (representative of 3 independent experiments). (H) Representative immunofluorescence for TMPRSS2 and (I) quantification of fluorescence intensity of TMPRSS2 (green) in TT1 cell line either stimulated or not (NS) for 24 hrs with IL1ß $(50 \mathrm{ng} / \mathrm{mL})$. Nuclei were counterstained with Hoechst 33342 (blue) and actin was stained with Phalloidin TexasRed (Red). CTCF, corrected total cell fluorescence. Scale bar: $10 \mu \mathrm{m} ; \mathrm{n}=3$ independent experiments. (J) Western blot analysis of TMPRSS2 in total protein lysates from $\Pi 1$ cells stimulated or not (NS) for 24 hours with IL $1 \beta$ (15 or $50 \mathrm{ng} / \mathrm{mL}$ ); GRP75 was used as a loading control (representative of 3 independent experiments). RTqPCR analysis of the relative gene expression of TMPRSS2 in (K) A549 or (L) TT1 cells stimulated for 4 hours with IL1 3 (50 $\mathrm{ng} / \mathrm{mL})$ and either treated or untreated with Birb796 (p38 MAPK inhibitor, 100 nM) or K7174 (GATA2 inhibitor, $10 \mu \mathrm{M}$ ). NS, Not Stimulated; $n=5$ independent experiments. (M) ChIP-RTqPCR analysis of the fold enrichment of GATA2 binding to the TMPRSS2 enhancer promotor region, in A549 cells either treated or untreated with Birb796 (p38 MAPK inhibitor, 100 nM) or K7174 (GATA2 inhibitor, $10 \mu \mathrm{M}$ ) and stimulated or not (NS) for 4 hours with IL1 $\beta$ (50 $\mathrm{ng} / \mathrm{mL})$. Data were adjusted by input for each sample and normalized with IgG (Mock). The fold enrichment of GATA2 binding to the IL1B and to the IL8 promotors was respectively used as positive and negative control. IgG was used as antibody specificity control. $\mathrm{N}=3$ independent experiments. (N) RT-qPCR analysis of the relative gene expression of TMPRSS2 in A549 cells upon GATA2 silencing with or without stimulation with IL $1 \beta(50 \mathrm{ng} / \mathrm{mL}$ ) for 4 hours. Scramble siRNA (Scr.) was used as a negative control; $n=4$ independent experiments.

(O) Representative confocal images and (P) quantification of fluorescence intensity of SARS-CoV-2 Spike protein (green) in A549 cells upon infection with heatinactivated SARS-CoV-2 virus. Cells were stimulated or not (NS) for 8 hours with IL1 $13(50 \mathrm{ng} / \mathrm{mL}$ ) and either treated or not with Birb796 (p38 MAPK inhibitor, 100 nM) or K7174 (GATA2 inhibitor, $10 \mu M$ ). Nuclei were counterstained in blue (Hoechst 33342). Scale bar: $10 \mu \mathrm{m} ; \mathrm{n}=3$ independent experiments. (Q) Representative confocal images and $(\mathbf{R})$ quantification of fluorescence intensity of SARS-CoV-2 Spike protein (green) in $\pi 1$ cells upon infection with heat-inactivated SARS-CoV-2 virus. Cells were stimulated or not (NS) for 8 hours with IL $1 \beta(50 \mathrm{ng} / \mathrm{mL}$ ) and either treated or not with Birb796 (p38 MAPK inhibitor, 100 nM) or K7174 (GATA2 inhibitor, $10 \mu \mathrm{M}$ ). Nuclei were counterstained in blue (Hoechst 33342). Scale bar: $10 \mu \mathrm{m} ; \mathrm{n}=3$ independent experiments. Data are presented as means \pm SEM. nonparametric Mann-Whitney $U$ test $(\mathbf{C}, \mathbf{D}, \mathbf{F}, \mathbf{I})$ or Kruskal-Wallis test for multiple comparisons with Dunn's post hoc $(\mathbf{A}, \mathbf{B}, \mathbf{K}, \mathbf{L}, \mathbf{M}, \mathbf{N}, \mathbf{P}, \mathbf{R}){ }^{*} \mathrm{P}<0.05,{ }^{* *} \mathrm{P}<0.01$, ${ }^{* \star \star} \mathrm{P}<0.001,{ }^{\star \star \star \star} \mathrm{P}<0.0001$. 
storm syndrome in COVID-19 patients $(15,16)$. Future high throughput studies should be performed to better understand the interconnection between IL1 $\beta$ and innate immune response in SARS-CoV-2 infection.

Collectively, our results indicated that the host inflammatory milieu might favor SARS-CoV-2 infection by increasing TMPRSS2 expression. In addition, we identified the molecular pathway that regulates this process that can be therapeutically targeted. Our findings unveiled a novel pathogenetic mechanism that associates the pro-inflammatory cytokine IL1 $\beta$ with an increased susceptibility to SARS-CoV-2 infection. Importantly, co-downregulation of $I L 1 B$ and TMPRSS2 upon azithromycin treatment was recently described in basal nasal epithelial cells, further suggesting a link between inflammation and protease expression in SARS-CoV-2 infection (17). Thus, targeting IL1 $\beta$ may not only reduce high inflammatory responses in severe COVID-19 patients, but also limit the viral entry in lung epithelial cells.

\section{METHODS}

\section{Participants, Study Design, and Data Collection}

Peripheral Blood (PB) was collected in EDTA tubes from enrolled controls and 44 COVID-19 patients that were admitted to the Infectious and Tropical Disease Unit of the University Hospital of Padua. Plasma was obtained after peripheral blood mononuclear cells (PBMC) isolation by density-gradient sedimentation using Ficoll-Paque PLUS (GE Healthcare, Germany) according to the manufacturer's protocol. Then, plasma was carefully removed from the $2 / 3$ of the top layer using a sterile serological pipette until the mononuclear cell interphase and stored at $-80^{\circ} \mathrm{C}$ until the analysis. The median age of the patients was 59.3 years (98-25). All patients were clinically diagnosed with COVID-19 (at least one positive laboratory PCR test for SARS-CoV-2 infection). All patients were classified into mild, moderate, severe, and critical cases based on results from chest imaging, clinical examination, and symptoms (WHO guidelines). The study was performed according to the ethical guidelines of the Declaration of Helsinki (7th revision). The study was approved by the Ethics Committee and the general authorization issued by the Data Protection Authority. Cod CESC n. 4933/AO/20.

\section{IL1 $\beta$ Quantification}

IL1 $\beta$ level was analyzed in the plasma from 4 controls and 44 patients by Luminex assay (Millipore, Billerica, USA). The diluted standard and quality control were used according to the manufacturer's instructions. The plate was read on Luminex $200^{\mathrm{TM}}$. Analysis was performed using xPONENT 3.1 software.

\section{Cell Culture and Treatments}

Human A549 cell line was donated from ECSIN lab, Padua. The cells were maintained in DMEM high glucose medium supplemented with $1 \%$ Na-pyruvate, 1\% L-glutamine, $10 \%$
Gibco FBS, $1 \%$ Penicillin-Streptomycin at $37^{\circ} \mathrm{C}$ under $5 \% \mathrm{CO} 2$, while human alveolar epithelial cell line (TT1) were maintained as Montagner et al. reported (18). Cells were stimulated with recombinant human IL1 $\beta$, IL6 or TNF $\alpha$ (PeproTech) at the concentration of 1,15 or $50 \mathrm{ng} / \mathrm{mL}$ either in combination or not with the inhibitors BIRB $796(100 \mathrm{nM})$ or K-7174 $(10 \mu \mathrm{M})$ (Selleck Chemicals). Vehicle treated and non-stimulated cells were used as a control condition. The treatment medium was composed of DMEM high glucose supplemented with PenicillinStreptomycin and 2\% FBS Gibco. RNA extraction was performed after 4 hours of treatment, whereas protein extraction was performed after 24 hours of treatment.

\section{Silencing}

Cells were transfected with GATA2 Stealth siRNA or scramble Silencer Select Negative Control (Thermo Fisher Scientific), according to the manufacturer's protocol. Briefly, cells were plated $24 \mathrm{~h}$ hours before transfection in a growth medium without antibiotics. Stealth siRNA - Lipofectamin 2000 (Invitrogen) complexes were prepared according to the manufacturer's instructions. Both siRNA and scramble were used at the concentration of 75 pmol. 24 hours after transfection cells were stimulated with recombinant human IL1 $\beta$ as previously described. RNA extraction was performed after 4 hours of treatment.

\section{RNA Extraction, Reverse Transcription, and RT-qPCR}

Total RNA was extracted for RT-qPCR analysis from unstimulated and stimulated cells with TRIzol reagent (Thermo Fisher Scientific) following manufacturer instructions. $500 \mathrm{ng}$ of RNA were retrotranscribed using High-Capacity cDNA Reverse Transcription Kit (Thermo Fisher Scientific) according to the manufacturer instructions. cDNA was diluted 1:10 and amplified with specific primers for the human genes TMPRSS2, ACE2, GATA2, FURIN, ADAM17 and GAPDH (Table 1) using Power SYBR Green Master Mix (Thermo Fisher Scientific) on a QuantStudio ${ }^{\text {TM }} 5$ Real-Time PCR System, 384-well (Applied Biosystems). The analysis of the obtained results was performed by the $\Delta \Delta \mathrm{Ct}$ method.

For the evaluation of cell susceptibility to SARS-CoV-2 infection, concerning the RNA extraction, we followed the protocol by Vogels et al. (19). We then performed reverse transcription as described above and RT-qPCR as described by WHO (20).

\section{Chromatin Immunoprecipitation}

Chromatin immunoprecipitation was performed with MAGnify ${ }^{\mathrm{TM}}$ Chromatin Immunoprecipitation System (Thermo Fisher Scientific) according to the manufacturer's protocol. Briefly, cells were plated and after 24 hours they were starved for 1 hour; then they were stimulated or not with recombinant $\operatorname{IL1} \beta(50 \mathrm{ng} / \mathrm{mL})$ and either treated or not with the indicated inhibitors in presence of IL1 $\beta(50 \mathrm{ng} / \mathrm{mL})$. After 4 hours of treatment, the DNA-transcription factor crosslink was performed with 1\% formaldehyde (Sigma Aldrich) in PBS for $15 \mathrm{~min}$ at RT in soft shaking; the reaction was stopped with 
TABLE 1 | Primers used in this study.

\begin{tabular}{|c|c|c|}
\hline & Forward & Reverse \\
\hline GAPDH & AACAGCCTCAAGATCATCAGC & GGATGATGTTCTGGAGAGCC \\
\hline TMPRSS2 & CCTCTAACTGGTGTGATGGCGT & TGCCAGGACTTCCTCTGAGATG \\
\hline ACE2 & TCCATTGGTCTTCTGTCACCCG & AGACCATCСАССТССАСТTСТС \\
\hline FURIN & GCCACATGACTACTCCGCAGAT & TACGAGGGTGAACTTGGTCAGC \\
\hline ADAM17 & AACAGCGACTGCACGTTGAAGG & CTGTGCAGTAGGACACGCCTा \\
\hline-13 kb TMPRSS2 & GCTGACCTITAATGAAGTITG & CCTAGTGAATITGGCCTCCTC \\
\hline IL1B promoter & TCGCACCCACTTCCTTCTCTT & TGCCAGAGGAAATGGTGACC \\
\hline IL8 promoter & GCTGAACCAGAGTTGGAACCC & GGTGCACTGGAGCTGCTTG \\
\hline nCoV_IP2 & ATGAGCTTAGTCCTGTTG & CTCCCTTGTTGTGTTGT \\
\hline IRF1 & TTGGCCTTCCACGTCTTG & GAGCTGGGCCATTCACAC \\
\hline IRF9 & ААСТGСССАСТСТССАСТTG & AGCCTGGACAGCAACTCAG \\
\hline ISG15 & GGCTTGAGGCCGTACTCC & СTGTTCTGGCTGACCTTCG \\
\hline IFITM2 & ATCCCGGTAACCCGATCAC & СПТСТGTСССТАGACTTСAC \\
\hline RNF20 & AAAGCATCGCACCATGTCTC & ATCCCACTGCAGGTCATCAA \\
\hline
\end{tabular}

glycine (Sigma) $125 \mathrm{mM}$ (5 min). Cells were then washed with PBS, scraped in 1,5 mL PBS and resuspended in 100uL of lysis buffer. Cells were then sonicated 20 times at $60 \mathrm{~Hz}$ for 20 seconds. To enrich GATA2 DNA binding sites, chromatin was incubated overnight with $1 \mu \mathrm{g}$ of GATA2 polyclonal antibody (GeneTex, cat\#GTX113441). Rabbit IgG was used as an antibody specificity control. DNA was purified using kit providedmagnetic beads. Fold enrichment was calculated following the manufacturer's instructions after qPCR amplification with Power SYBR Green Master Mix (Thermo Fisher Scientific) on a QuantStudio $^{\text {TM }} 5$ Real-Time PCR System. Reported specific primers for -13kb TMPRSS2, IL1B,IL8, nCoV_IP2, IRF1, IRF9, ISG15, IFITM2 and RNF20 were used (Table 1) (20-24).

\section{Protein Extraction and Western Blot}

Protein lysates from A549 or TT1 cells were prepared using a lysis buffer (10 mM Tris-HCl pH 9, 4\% SDS, 1 mM DTT). $5 \mu \mathrm{g}$ of total protein extracts were loaded for western blot in Bolt ${ }^{\mathrm{TM}} 4$ 12\% Bis-Tris Plus gels (Thermo Fisher Scientific) and blotted onto a PVDF membrane (Bio-Rad). Membranes were blocked with $3 \%$ bovine serum albumin (Sigma Aldrich) for 1 hour and then incubated with the primary antibodies $\alpha$-TMPRSS2 antibody (EPR3862, Abcam) or $\alpha$-GRP75 (D-9, Santa Cruz Biotechnology) at $4^{\circ} \mathrm{C}$. After that, membranes were incubated with the secondary antibody goat $\alpha$-rabbit $\operatorname{IgG}(\mathrm{H}+\mathrm{L})$-HRP Conjugate (Bio-Rad) for 1 hour. Chemiluminescence was detected with iBright 1500 (Invitrogen). Images were analyzed with ImageJ software (Fiji) and the protein was quantified and normalized on the housekeeping protein (GRP75) and on the non-stimulated condition.

\section{Immunofluorescence Analysis}

For TMPRSS2 protein immunofluorescence, A549 and TT1 cells were either stimulated or not with IL1 $\beta$ for 24 hours. By contrast, for the evaluation of cell susceptibility to SARS-CoV-2 infection, cells were either stimulated or not with IL1 $\beta$ and the selected inhibitors for 8 hours as previously described. The infection was then performed, using Heat-inactivated SARS-CoV-2 (VR$1986 \mathrm{HK}, \mathrm{ATCC})$ at 4 TCID50/mL. 72 hours after the infection, cells were fixed with PFA 4\% (Sigma) in PBS, blocked with blocking solution (PBS, 1\% BSA, 0,02\% NP40) and incubated with SARS-CoV-2 spike antibody (polyclonal, GeneTex) followed by anti-rabbit Alexa Fluor 488 antibody (polyclonal, Invitrogen). Nuclei were counterstained with Hoechst 33342 (Invitrogen) and actin was stained with Phalloidin TexasRed (Invitrogen). Fluorescence images were acquired using the Zeiss LSM 800 confocal laser scanning microscope. The fluorescent signal per cell was quantified using ImageJ software (Fiji) and the corrected total cell fluorescence (CTCF) was calculated. TMPRSS2 staining in A549 and TT1 cells was performed by using H-4 (Santa Cruz Biotechnology) antibody according to the indicated protocol. $3 \mathrm{D}$ reconstruction was performed using ZEN 3.2 Blue edition software (Carl Zeiss).

\section{Statistical Analysis}

Data are reported as the mean \pm SEM of at least 3 independent experiments. Statistical analysis was performed using GraphPad Prism 6 (GraphPad Software). Comparisons between two groups were performed by the nonparametric Mann-Whitney $U$ test or Kruskal-Wallis test for multiple comparisons with Dunn's post hoc test ${ }^{*} \mathrm{P}<0.05,{ }^{* *} \mathrm{P}<0.01,{ }^{* * *} \mathrm{P}<0.001,{ }^{* * * *} \mathrm{P}<0.0001$.

\section{DATA AVAILABILITY STATEMENT}

The raw data supporting the conclusions of this article will be made available by the authors, without undue reservation.

\section{ETHICS STATEMENT}

The studies involving human participants were reviewed and approved by the Ethics Committee and the general authorization issued by the Data Protection Authority. Cod CESC n. 4933/AO/ 20. The patients/participants provided their written informed consent to participate in this study. 


\section{AUTHOR CONTRIBUTIONS}

Conceptualization, AV, RS-R and BM. Investigation CC, FCV, RA, NB, FM, AC and RS-R. Writing- original draft, RS-R, CC and BM. Critical data discussion, AV, BM and RS-R. Funding acquisition AV. All authors contributed to the article and approved the submitted version.

\section{FUNDING}

The study was funded by Fondazione Città della Speranza, grant number 20/02CoV, and Fondazione Cassa di Risparmio di Padova e Rovigo (CARIPARO, Bando Progetti di Ricerca Covid 2019, $\mathrm{n}^{\circ}$ 55784) to AV.

\section{ACKNOWLEDGMENTS}

We thank all the patients who participated to the study and the entire clinical staff of the Infectious Disease Unit of the Padova University Hospital. We thank Diletta Arcidiacono, Alice Zaramella and Stefano Realdon for LUMINEX assay.

\section{SUPPLEMENTARY MATERIAL}

The Supplementary Material for this article can be found online at: https://www.frontiersin.org/articles/10.3389/fimmu.2021.781352/ full\#supplementary-material

Supplementary Figure 1 | Role of IL1 $\beta$, IL6, and TNF $\alpha$ in SARS-CoV-2 receptors expression. RT-qPCR analysis of the relative gene expression of (A) TMPRSS2 in A549 cells either stimulated or not (NS) for 4 hours with IL6 or TNF $\alpha$ (15 or $50 \mathrm{ng} /$ $\mathrm{mL}) ; \mathrm{n}=5$ independent experiments; (B) ACE2 in A549 cell line either stimulated or not (NS) for 4 hours with IL6 or TNF $\alpha$ ( 15 or $50 \mathrm{ng} / \mathrm{mL}$ ); $\mathrm{n}=3$ independent experiments. ACE2 expression in (C) A549 or (D) TT1 cells either stimulated or not (NS) for 4 hours with IL1 $\beta$. Data are presented as means \pm SEM. Nonparametric Mann-Whitney $U$ test. ${ }^{* \star *} P<0.0001,{ }^{* *} P<0.01$.

Supplementary Figure 2 | ADAM17 and FURIN expression was not transcriptionally modulated by inflammatory cytokines. RT-qPCR analysis of the relative gene expression of (A) ADAM17 and (B) FURIN in A549 cell line either

\section{REFERENCES}

1. Angioni R, Sánchez-Rodríguez R, Munari F, Bertoldi N, Arcidiacono D, Cavinato S, et al. Age-Severity Matched Cytokine Profiling Reveals Specific Signatures in Covid-19 Patients. Cell Death Dis (2020) 11:1-12. doi: 10.1038/ s41419-020-03151-Z

2. Zhou Y, Yang Q, Chi J, Dong B, Lv W, Shen L, et al. Comorbidities and the Risk of Severe or Fatal Outcomes Associated With Coronavirus Disease 2019: A Systematic Review and Meta-Analysis. Int J Infect Dis (2020) 99:47-56. doi: 10.1016/j.ijid.2020.07.029

3. McElvaney OJ, McEvoy NL, McElvaney OF, Carroll TP, Murphy MP, Dunlea DM, et al. Characterization of the Inflammatory Response to Severe COVID-19 Illness. Am J Respir Crit Care Med (2020) 202:812-21. doi: 10.1164/rccm.202005-1583OC

4. Yap JKY, Moriyama M, Iwasaki A. Inflammasomes and Pyroptosis as Therapeutic Targets for COVID-19. J Immunol Baltim Md 1950 (2020) 205:307-12. stimulated or not (NS) for 4 hours with IL1 $\beta$, IL6 or TNF $\alpha$ (15 or 50 ng/mL); n=3 independent experiments. Data are presented as means \pm SEM.

Supplementary Figure 3 | TMPRSS2 expression in response to IL1 $\beta$ stimulation in A549 cells. Quantification of western blots for TMPRSS2 protein in (A) A549 or (B) $T 11$ cells stimulated or not with IL1 $\beta$ (15 or $50 \mathrm{ng} / \mathrm{mL}$ ) for 24 hours. Data are presented as means \pm SEM. Nonparametric Mann-Whitney $U$ test. ${ }^{\star} P<0.05$. (C) $3 \mathrm{D}$ reconstruction of TMPRSS2 (green) immunofluorescent confocal images in A549 and TT1 cells either stimulated or not (NS) with IL $1 \beta$ (50 ng/mL). Nuclei were counterstained with Hoechst 33342 (blue) and actin was stained with Phalloidin TexasRed (Red), $\mathrm{n}=3$ independent experiments.

Supplementary Figure 4 | IL1 $\beta$ did not affect transcriptionally endosomal and replication genes for SARS-CoV-2. RT-qPCR analysis of the relative gene expression of (A, B) IFITM2 and (C, D) RNF20 in A549 and T11 cell line either stimulated or not (NS) for 4 hours with IL1 $\beta$ (50 ng/mL); $n=3$ independent experiments. Data are presented as means \pm SEM.

Supplementary Figure 5 | IL1 $\beta$ and selected inhibitors did not affect ACE2 expression in A549 cell line and the inhibitors vehicle did not alter the observed results. (A) RT-qPCR analysis of the relative gene expression of TMPRSS2 in A549 cell line either stimulated or not (NS) with IL1 $\beta(50 \mathrm{ng} / \mathrm{mL})$ for 4 hours in presence or not of DMSO (Vehic. of the inhibitors). (B) RT-qPCR analysis of the relative gene expression of ACE2 in A549 cell line either stimulated or not (NS) with IL1 $\beta$ (50 ng/

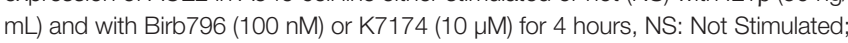
$\mathrm{n}=4$ independent experiments. Data are presented as means \pm SEM.

Supplementary Figure 6 | GATA2 expression is not affected by inflammatory cytokine stimulation. RT-qPCR analysis of the relative gene expression of GATA2 in (A) A549 or (B) TT1 cells are either stimulated or not for 4 hours with IL1 $\beta$ (15 or 50 $\mathrm{ng} / \mathrm{mL}$ ); NS: Not Stimulated; $\mathrm{n}=3$ independent experiments. Data are presented as means \pm SEM.

Supplementary Figure 7 | GATA2 silencing controls and Spike immunofluorescence negative controls. Relative gene expression of (A) GATA2 and (B) E-Cadherin after GATA2 silencing in A549 cell line. (C) Representative western blot for GATA2 and quantification after silencing in A549 cell line. Scramble siRNA was used as a control. $n=3$ independent experiments. (D) Representative confocal images of A549 and $\Pi 1$ cells without infection with heat-inactivated SARS-CoV-2 virus. (E) Relative gene expression of $\mathrm{nCoV}$ _IP2 upon infection with heat-inactivated SARS-CoV-2 virus at different time points (2, 24, and 48 hours). Before infection, cells were stimulated or not (NS) for 8 hours with $\mathrm{IL} 1 \beta(50 \mathrm{ng} / \mathrm{mL})$ and either treated or not with Birb796 (p38 MAPK inhibitor, 100 nM) or K7174 (GATA2 inhibitor, 10 $\mu \mathrm{M})$. Data are presented as means \pm SEM. Nonparametric Kruskal-Wallis test for multiple comparisons with Dunn's post hoc. ${ }^{*} P<0.05^{\star *} \mathrm{P}<0.01{ }^{\star \star *} \mathrm{P}<0.001$.

Supplementary Figure 8 | Effect of IL1 $\beta$ over interferon response genes. Relative gene expression for (A, B) IRF1 and (C, D) ISG15 and (E, F) IRF9 in A549 and TT1 cell line either stimulated or not (NS) for 4 hours with IL1 $\beta(50 \mathrm{ng} / \mathrm{mL}) ; \mathrm{n}=3$ independent experiments. Data are presented as means \pm SEM. Nonparametric Mann-Whitney $U$ test. ${ }^{\star \star} \mathrm{P}<0.01,{ }^{\star \star \star *} \mathrm{P}<0.0001$.

5. Hoffmann M, Kleine-Weber H, Schroeder S, Krüger N, Herrler T, Erichsen S et al. SARS-CoV-2 Cell Entry Depends on ACE2 and TMPRSS2 and Is Blocked by a Clinically Proven Protease Inhibitor. Cell (2020) 181:271-80.e8. doi: 10.1016/j.cell.2020.02.052

6. Sungnak W, Huang N, Bécavin C, Berg M, Queen R, Litvinukova M, et al. SARS-CoV-2 Entry Factors are Highly Expressed in Nasal Epithelial Cells Together With Innate Immune Genes. Nat Med (2020) 26:681-7. doi: 10.1038/s41591-020-0868-6

7. Ziegler CGK, Allon SJ, Nyquist SK, Mbano IM, Miao VN, Tzouanas CN, et al. SARS-CoV-2 Receptor ACE2 Is an Interferon-Stimulated Gene in Human Airway Epithelial Cells and Is Detected in Specific Cell Subsets Across Tissues. Cell (2020) 181:1016-35.e19.

8. Clinckemalie L, Spans L, Dubois V, Laurent M, Helsen C, Joniau S, et al. Androgen Regulation of the TMPRSS2 Gene and the Effect of a SNP in an 
Androgen Response Element. Mol Endocrinol (2013) 27:2028-40. doi: 10.1210/me.2013-1098

9. Zipeto D, Palmeira J, da F, Argañaraz GA, rgañaraz ER. ACE2/ADAM17/ TMPRSS2 Interplay May Be the Main Risk Factor for COVID-19. Front Immunol (2020) 11:2642. doi: 10.3389/fimmu.2020.576745

10. Prelli Bozzo C, Nchioua R, Volcic M, Koepke L, Krüger J, Schütz D, et al. IFITM Proteins Promote SARS-CoV-2 Infection and are Targets for Virus Inhibition In Vitro. Nat Commun (2021) 12:4584. doi: 10.1038/s41467-021-24817-y

11. Zhang S, Wang J, Cheng G. Protease Cleavage of RNF20 Facilitates Coronavirus Replication via Stabilization of SREBP1. Proc Natl Acad Sci (2021) 118:37-44. doi: 10.1073/pnas.2107108118

12. Katsumura KR, Ong IM, DeVilbiss AW, Sanalkumar R, Bresnick EH. GATA Factor-Dependent Positive-Feedback Circuit in Acute Myeloid Leukemia Cells. Cell Rep (2016) 16:2428-41. doi: 10.1016/j.celrep.2016.07.058

13. Aarreberg LD, Esser-Nobis K, Driscoll C, Shuvarikov A, Roby JA, Gale M Jr, et al. Interleukin- $1 \beta$ Induces mtDNA Release to Activate Innate Immune Signaling via cGAS-STING. Mol Cell (2019) 74:801-15.e6. doi: 10.1016/ j.molcel.2019.02.038

14. Li X-Q, Li XN, Liang J-J, Cai X-B, Tao Q, Li Y-X, et al. IRF1 Up-Regulates Isg15 Gene Expression in dsRNA Stimulation or CSFV Infection by Targeting Nucleotides -487 to -325 in the 5' Flanking Region. Mol Immunol (2018) 94:153-65. doi: 10.1016/j.molimm.2017.12.025

15. Munnur D, Teo Q, Eggermont D, Lee HHY, Thery F, Ho J, et al. Altered ISGylation Drives Aberrant Macrophage-Dependent Immune Responses During SARS-CoV-2 Infection. Nat Immunol (2021) 22:1416-27. doi: 10.1038/s41590-021-01035-8

16. Cao X. ISG15 Secretion Exacerbates Inflammation in SARS-CoV-2 Infection. Nat Immunol (2021) 22:1360-2. doi: 10.1038/s41590-021-01056-3

17. Renteria AE, Endam LM, Adam D, Filali-Mouhim A, Maniakas A, Rousseau S, et al. Azithromycin Downregulates Gene Expression of IL-1 $\beta$ and Pathways Involving TMPRSS2 and TMPRSS11D Required by SARS-CoV-2. Am J Respir Cell Mol Biol (2020) 63:707-9. doi: 10.1165/rcmb.2020-0285LE

18. Montagner M, Bhome R, Hooper S, Chakravarty P, Qin X, Sufi J, et al. Crosstalk With Lung Epithelial Cells Regulates Sfrp2-Mediated Latency in Breast Cancer Dissemination. Nat Cell Biol (2020) 22:289-96. doi: 10.1038/ s41556-020-0474-3

19. Vogels CBF, Watkins AE, Harden CA, Brackney DE, Shafer J, Wang J, et al. SalivaDirect: A Simplified and Flexible Platform to Enhance SARS-CoV-2 Testing Capacity. Med N Y N (2021) 2:263-80.e6.
20. WHO Real-Time RT-PCR Assays for the Detection of SARS-CoV-2 Institut Pasteur, Paris. Available at: https://www.who.int/docs/default-source/ coronaviruse/real-time-rt-pcr-assays-for-the-detection-of-sars-cov-2institut-pasteur-paris.pdf?sfvrsn=3662fcb6_2 (Accessed on 14 October 2021).

21. Robichon K, Maiwald T, Schilling M, Schneider A, Willemsen J, Salopiata F, et al. Identification of Interleukin $1 \beta$ as an Amplifier of Interferon AlphaInduced Antiviral Responses. PLoS Pathog (2020) 16:e1008461. doi: 10.1371/ journal.ppat.1008461

22. Xu-yang Z, Pei-Yu B, Chuan-Tao Y, Wei Y, Hong-Wei M, Kang T, et al. Interferon-Induced Transmembrane Protein 3 Inhibits Hantaan Virus Infection, and Its Single Nucleotide Polymorphism Rs12252 Influences the Severity of Hemorrhagic Fever With Renal Syndrome. Front Immunol (2017) 7:535. doi: 10.3389/fimmu.2016.00535

23. Linnemann AK, O'Geen H, Keles S, Farnham PJ, Bresnick EH. Genetic Framework for GATA Factor Function in Vascular Biology. Proc Natl Acad Sci USA (2011) 108:13641-6. doi: 10.1073/pnas.1108440108

24. Wang D, Wang Y, Wu X, Kong X, Li J, Dong C. RNF20 Is Critical for SnailMediated E-Cadherin Repression in Human Breast Cancer. Front Oncol (2020) 10:2762. doi: 10.3389/fonc.2020.613470

Conflict of Interest: The authors declare that the research was conducted in the absence of any commercial or financial relationships that could be construed as a potential conflict of interest.

Publisher's Note: All claims expressed in this article are solely those of the authors and do not necessarily represent those of their affiliated organizations, or those of the publisher, the editors and the reviewers. Any product that may be evaluated in this article, or claim that may be made by its manufacturer, is not guaranteed or endorsed by the publisher.

Copyright (c) 2021 Cioccarelli, Sánchez-Rodríguez, Angioni, Venegas, Bertoldi, Munari, Cattelan, Molon and Viola. This is an open-access article distributed under the terms of the Creative Commons Attribution License (CC BY). The use, distribution or reproduction in other forums is permitted, provided the original author(s) and the copyright owner(s) are credited and that the original publication in this journal is cited, in accordance with accepted academic practice. No use, distribution or reproduction is permitted which does not comply with these terms. 